\title{
Accuracy of noninvasive blood pressure and cardiac output measurement during living kidney transplantation
}

Tadayuki Ishibashi, Kengo Hayamizu, Kentaro Tokuda, Yuji Karashima, Sumio Hoka

Department of Anesthesiology and Critical Care Medicine, Kyushu University Hospital, Japan

\section{$<$ Background and Goal of study>}

Perioperative low cardiac output (CO) and instability of blood pressure (BP) have been shown to be associated with poor clinical outcome in living kidney transplantation recipients.

A new cardiovascular monitoring system based on the volume-clamp method (ClearSight ${ }^{\mathrm{TM}}$; Edwards Lifesciences Corp., Irvine, CA, USA), was developed to monitor the beat-to-beat BP, stroke volume (SV) and continuous CO (CCO) noninvasively. We assessed the accuracy of BP and CO measured by the ClearSight ${ }^{\mathrm{TM}}$ system, compared to an invasive monitoring system (FloTrac ${ }^{\mathrm{TM}}$; Edwards Lifesciences Corp.) during living kidney transplantation.

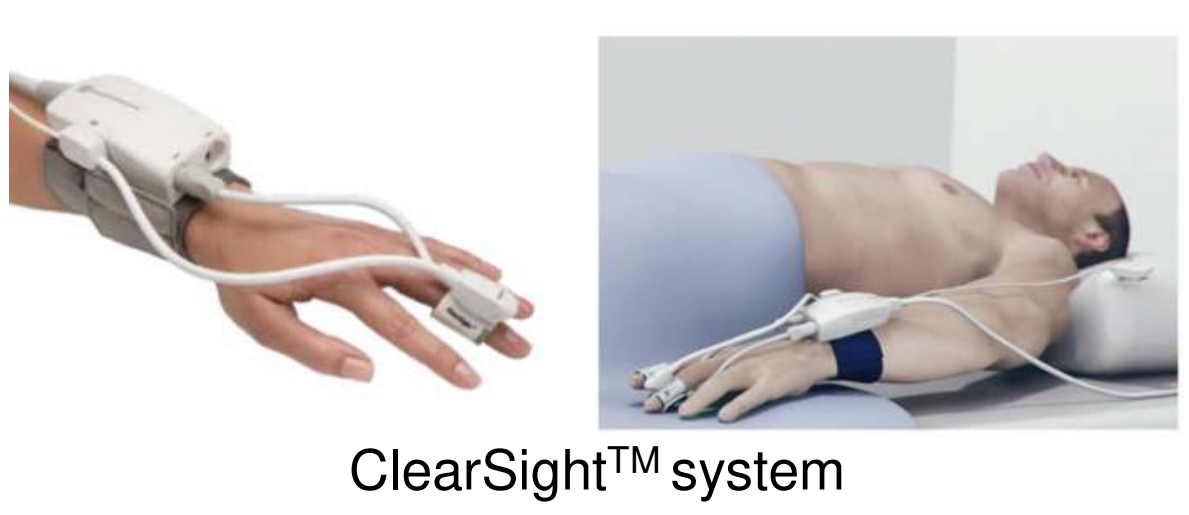

$<$ Material and Methods>
$<$ Volume-clamp method>

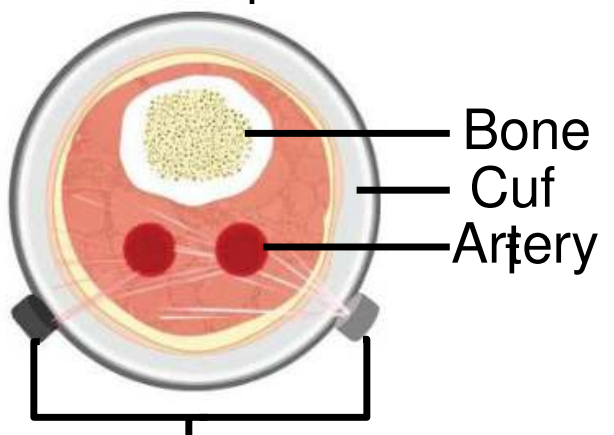

Photo-plethysmographic system
$<$ Physiocal method>

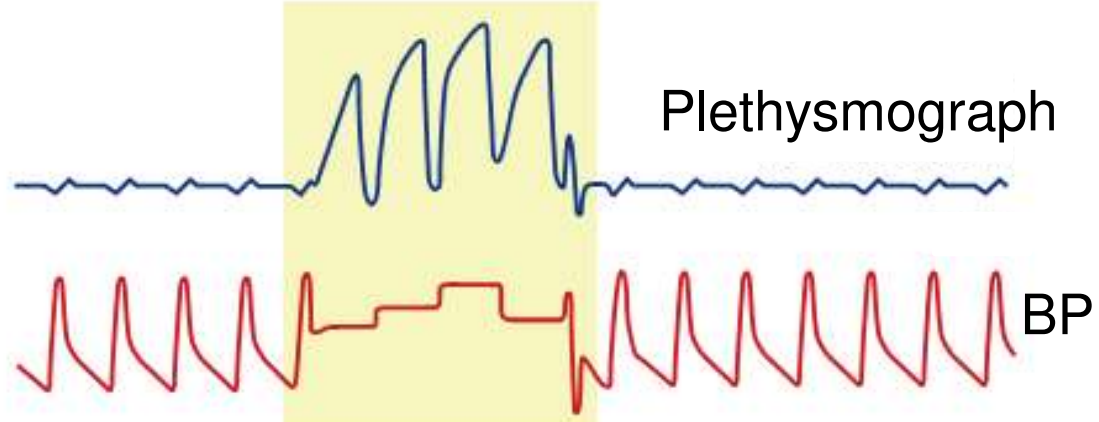

Two recipients undergoing kidney transplantation were included in this study. We extracted the value of mean arterial pressure(MAP) and CO every 3 minutes, simultaneously using FloTrac ${ }^{\mathrm{TM}}\left(\mathrm{MAP}_{\mathrm{FT}}\right.$ and $\mathrm{CO}_{\mathrm{FT}}$, respectively) and $\mathrm{ClearSight}^{\mathrm{TM}}$ (MAP $_{C S}$ and $\mathrm{CO}_{\mathrm{CS}}$, respectively). In total, we obtained 197 points of consecutive data from 2 patients (patient1: 90 points, patient2: 107 points). Data were statistically analyzed by using the Spearman's rank-correlation coefficient and the BlandAltman analysis.

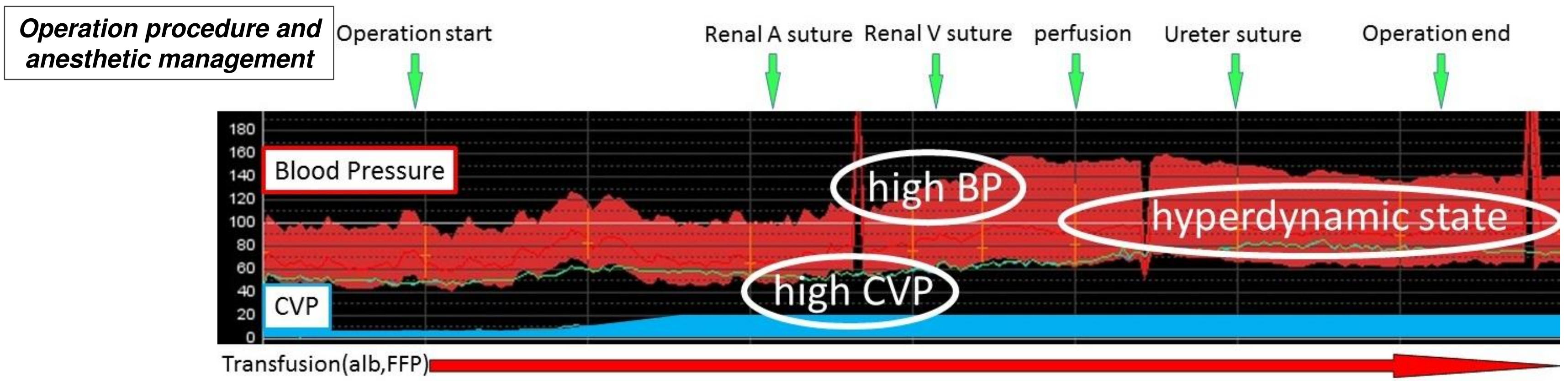

Catecholamine(DOA,NAd)

$<$ Results and Discussion>

MAP

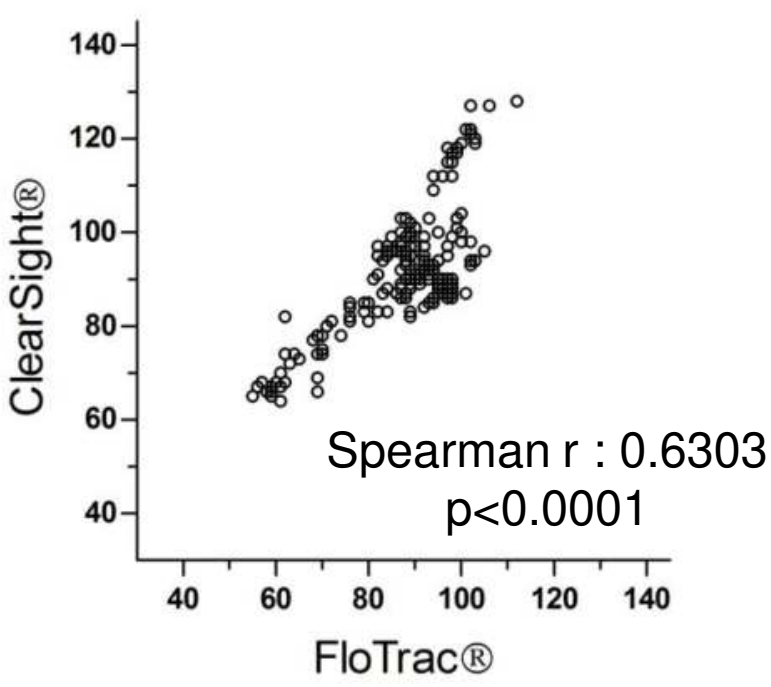

$\mathrm{CO}$

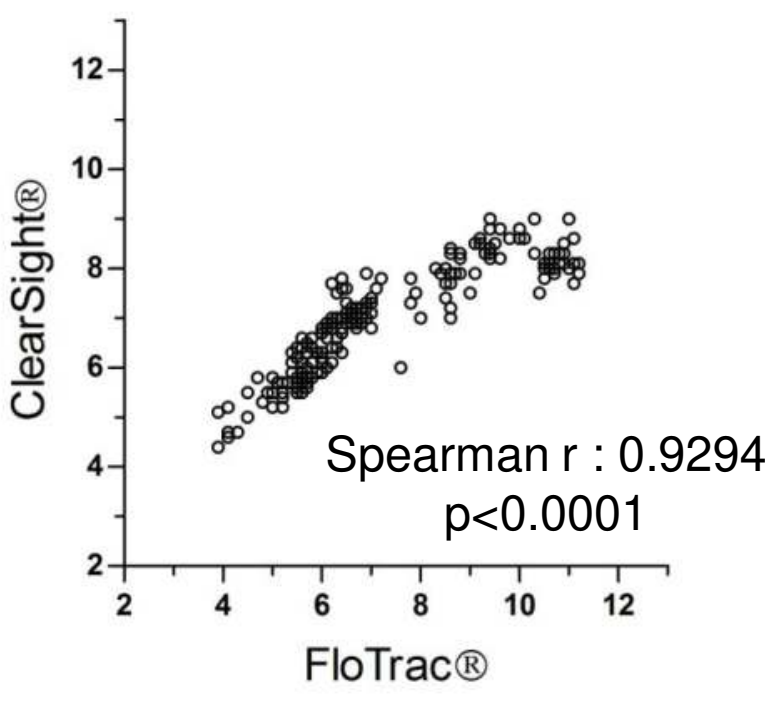

Bland-Altman plot of MAP : Difference vs average

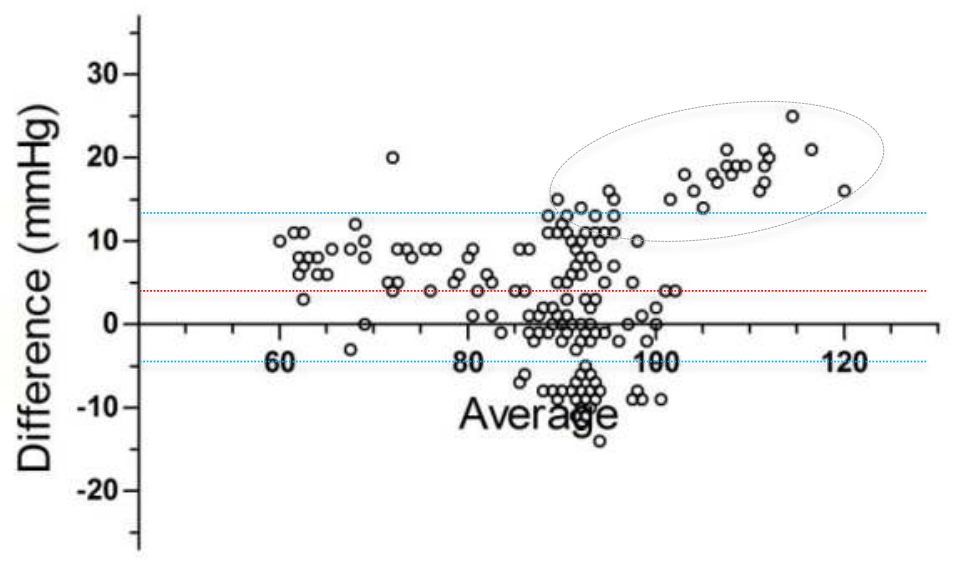

Agreement : $4.51 \pm 8.30 \mathrm{mmHg}$ and the mean percentage error was $18.98 \%$

Bland-Altman plot of CO : Difference vs average

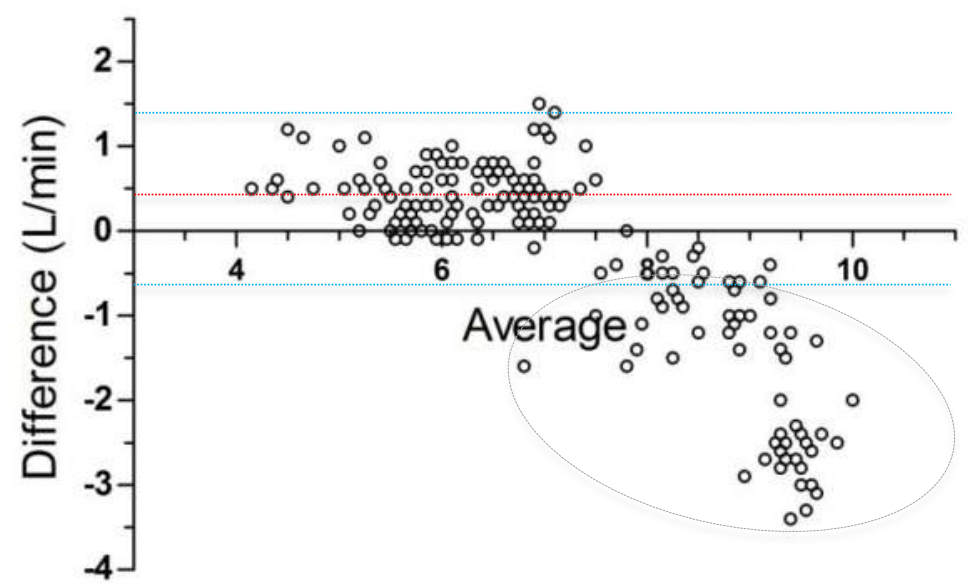

Agreement : $0.23 \pm 1.14 \mathrm{~L} / \mathrm{min}$ and the mean percentage error was $31.98 \%$
Actual trend (Case:1)

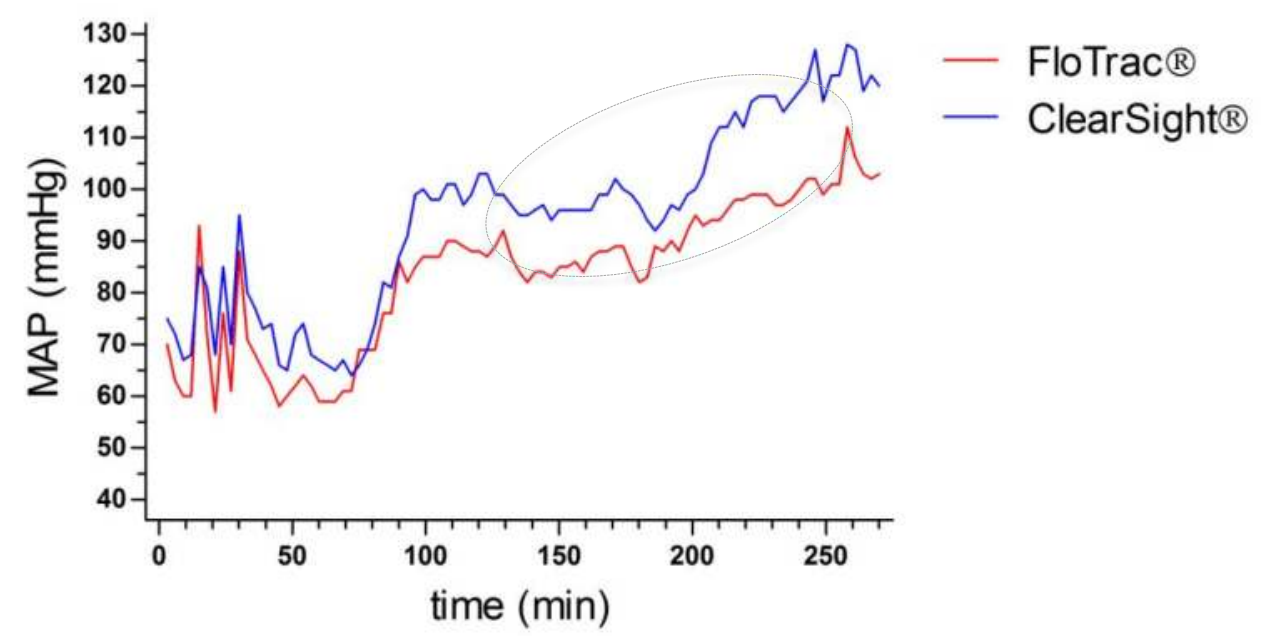

Actual trend (Case:1)

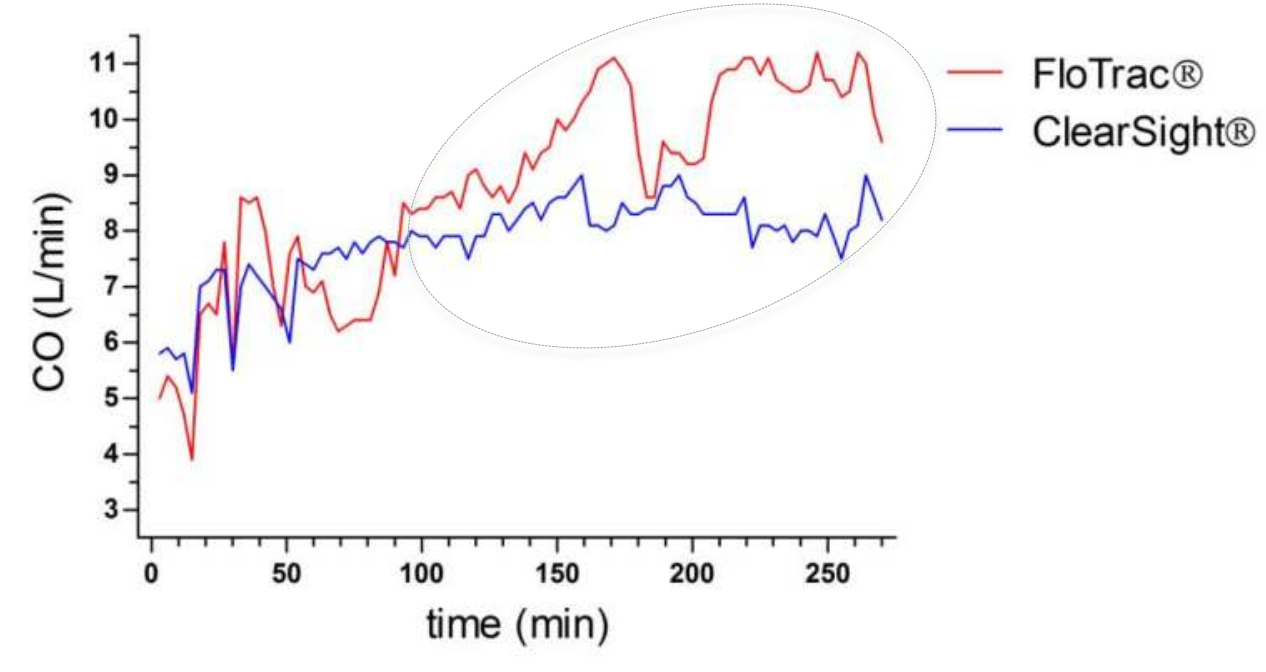

\section{$<$ Conclusion>}

The value of MAP and CO measured by ClearSight ${ }^{\mathrm{TM}}$ system and FloTrac ${ }^{\mathrm{TM}}$ are related to positive correlation statistically. However, parameters of ClearSight ${ }^{\mathrm{TM}}$ was unstable in the hyperdynamic state. Further research is needed to confirm ClearSight ${ }^{\mathrm{TM}}$ system can be reliable monitor during living kidney transplantation. 